\title{
Isolation and characterization of a strain of Lichtheimia corymbifera (ex Absidia corymbifera) from a case of bovine abortion
}

\author{
Chiara Piancastelli, Francesca Ghidini, Gaetano Donofrio, Stefano Jottini, \\ Simone Taddei, Sandro Cavirani and Clotilde S Cabassi*
}

Address: Dipartimento di Salute Animale, Parma University, via del Taglio 10, 43126 Parma, Italy

Email: Chiara Piancastelli - chiaravet@libero.it; Francesca Ghidini - francy1176@libero.it; Gaetano Donofrio - gaetano.donofrio@unipr.it; Stefano Jottini - stefano.jottini@gmail.com; Simone Taddei - simone.taddei@unipr.it; Sandro Cavirani - sandro.cavirani@unipr.it; Clotilde S Cabassi* - clotildesilvia.cabassi@unipr.it

* Corresponding author

Published: 30 November 2009

Reproductive Biology and Endocrinology 2009, 7:138 doi:10.1186/1477-7827-7-138

This article is available from: http://www.rbej.com/content/7/1//38

(c) 2009 Piancastelli et al; licensee BioMed Central Ltd.

This is an Open Access article distributed under the terms of the Creative Commons Attribution License (http://creativecommons.org/licenses/by/2.0), which permits unrestricted use, distribution, and reproduction in any medium, provided the original work is properly cited.

\begin{abstract}
Background: Lichtheimia corymbifera (previously Absidia corymbifera) is a filamentous zygomycetes belonging to the order Mucorales and to the family Lichtheimiaceae. Members of genus Lichtheimia spp. are cosmopolitan and ubiquitous in nature. Lichtheimia corymbifera is a recognized agent of diseases in man and animals. In cattle it causes abortion and mastitis. Three cases of bovine abortion occurred in a herd located in the Po Valley. Serological examinations were performed on fetal and mother's blood. One of the aborted fetus was referred to our laboratory. The paper describes the isolation and characterization of Lichtheimia corymbifera from a bovine aborted fetus.
\end{abstract}

Methods: Serological examinations were performed on fetal and mother's blood. Lesions on fetal tissues and placenta leaded the diagnostic suspect towards a mycotic aetiology. Tissues were then put in culture, and at the same time an histological examination was performed, together with bacteriological and virological tests. The isolate from placenta and fetal tissues was identified and characterized by PCR and RFLP, using the ITS region as a target sequence and Acll restriction site within the amplicon to distinguish Lichtheimia corymbifera among the other fungi.

Results: Serological, bacteriological and virological tests gave aspecific results. Histological examination evidenced numerous PAS positive hyphae within the necrotic cotiledons and numerous fungal nonseptate hyphae to the GMS stain. Colonies with typical morphological features of fungi grew up on Sabouraud agar from fetal skin and placenta. On the developed colonies the microscopic examination has shown a large number of nonseptate hyphae and sporangia consistent with Mucorales. PCR and RFLP allowed the identification of the isolate as Lichtheimia corymbifera.

Conclusion: The present report describes the isolation and the molecular characterisation of a fungal isolate from bovine aborted fetus and placenta. The diagnostic protocol allowed to identify and characterise the strain. This is the first isolation in Italy of Lichtheimia corymbifera in a bovine aborted fetus. 


\section{Background}

Bovine abortion is defined as an interruption of pregnancy between the $42^{\text {nd }}$ and $260^{\text {th }}$ day [1]. Among the infectious causes of abortion in cattle are included bacteriological, viral, parasitic and mycotic agents [2]. The knowledge of fungi as abortive pathogens is dated from the last century, and mycotic placentitis is a cause of abortion in cattle worldwide [3].

In the northern hemisphere the incidence of mycotic abortion is sporadic, occurring mainly during the winter periods (November - April), when the cows are usually fed with a large amount of hay [3]. This phenomenon is more evident in conditions of poor aeration and high humidity, which promotes the environmental growth of fungi [4]. The administration of wet stored and mouldy fodder, in which a large amount of fungal spores could be present, is a further risk factor for the mycotic abortion. The fungal spores can penetrate through gastric lesions or the respiratory tract and reach the placenta and the fetus where there are optimal conditions for their full development [5]. The mycotic abortion in cattle usually occurs at the $6^{\text {th }}-8^{\text {th }}$ months of gestation and is often followed by placenta retention. The placenta is thickened and wooden in appearance [6], and haemorrhagic necrotizing placentitis, often associated with thickened yellowish necrotic cotyledons may be present $[3,7]$. On the fetus skin lesions similar to parakeratotic dermatitis are usually evident, characterized by raised ringworm-like fungal skin plaques and blefaritis $[5,8,9]$.

Different fungi have been isolated from aborted fetuses, belonging to the species Aspergillus fumigatus and Aspergillus nidulans, Absidia corymbifera, Mortierella wolfii, Rhizopus spp., Mucor spp., and Rhizomucor spp. [4,10]. Previous reports recorded that $>60 \%$ of cases of abortion in the USA were caused by uncomplicated infection with Aspergillus fumigatus. Zygomycetes (Absidia, Mortierella, Rhizomucor, Rhizopus) accounted for about $20 \%$ of cases, and the remaining $20 \%$ were caused by a wide range of opportunistic filamentous fungi and yeasts [3]. In Australia Aspergillus spp. and Mortierella wolfii have been isolated [11], and in New Zealand Mortierella wolfii has been considered the major mycotic cause of abortion [12]. In Europe, there are reports of mycotic abortion in Great Britain due to Aspergillus, Mortierella and Absidia spp. since $1967[13]$ and more recently $[6,14]$. In Italy only cases of mycotic abortion due to Aspergillus spp. are reported [15].

In February 2009 three cases of abortion occurred during a single week in a Friesian dairy herd located in the province of Parma (Po Valley), area of production of Parmigiano Reggiano cheese. The herd size was 120 animals (60 lactating), free-stall housing, not vaccinated for infectious abortive agents. The abortions occurred between the $5^{\text {th }}$ and the $6^{\text {th }}$ month of gestation, without apparent symp- toms. Signs of illness were not observed in the dams before and after abortions. Of the three aborted fetuses, only one showed skin lesions. It was aborted at the $23^{\text {rd }}$ week of pregnancy and referred to our laboratory for diagnostic investigations. Aim of the work is to describe the isolation and characterisation of a strain of Lichtheimia corymbifera isolated from the referred fetus, to provide a complete diagnostic protocol in case of bovine mycotic abortion.

\section{Methods Samples}

The aborted fetus and placenta were chilled and referred to our laboratory within 12 hours from the abortion, together with the blood samples from the aborted cows.

\section{Histological examinations}

Histological examinations were performed on fetal tissue samples including skin, heart, lung, liver, spleen, kidney, fetal stomach and placenta collected during the necropsy and fixed in $4 \%$ buffered formalin (Bio-Optica). The samples were routinely processed and stained with hematoxylin and eosin (HE) for histopathological evaluation. To investigate the presence of fungi, periodic acid Schiff reaction (PAS) and Grocott's Methenamine Silver stain (GMS) were also performed on tissue sections.

\section{Culture}

Bacteriological examination was performed on fetal spleen, liver, kidney, lung, heart, skin, stomach content and placenta on blood agar (5\% bovine erythrocytes) and Mc Conkey agar (DIFCO) and incubated for 24-48 hours at $37^{\circ} \mathrm{C}$ in air. The fetal stomach content was specifically cultured for the isolation of Brucella spp. on 5\% blood agar and incubated in microaerophilic $\left(5 \% \mathrm{CO}_{2}\right)$ environment.

Mycological culture was performed on fetal spleen, liver, kidney, lung, heart and placenta on Sabouraud agar (DIFCO), and incubated for $24-48$ hours at $37^{\circ} \mathrm{C}$ in air.

\section{Determination of melezitose assimilation}

Melezitose assimilation was performed using ID32C strip (bioMérieux, Marcy l'Etoile, France) as reported by Schwarz and coll. [16].

\section{Serological examinations}

Serological examinations for the main infectious abortive agents were performed on blood sera from aborted cows. Moreover, during the necropsy of the fetus, $1 \mathrm{ml}$ of blood from the atrial chambers of the heart was taken. The serological examination of the blood samples to investigate the presence of antibodies against abortive agents was performed for Brucella abortus by card test agglutination (Brucelloslide Test, bioMérieux), Neospora caninum by indirect immunofluorescence, Chlamydophila abortus by ELISA 
(Civtest Bovis Chlamydia ps., Hipra), Bovine Viral Diarrea (BVD) virus by seroneutralization, Coxiella burnetii by ELISA (Chekit Q Fever antibody ELISA test kit, IDEXX), and Leptospira serovar sejroe-hardjo, australis-bratislava, pomona-pomona, icterohaemorrhagiae-copenhageni by microagglutination test. ELISAs were performed following manufacturer instructions. Seroneutralization, indirect immunofluorescence and microagglutination test were performed following our in-house protocols.

\section{Virological examination}

Virological examination was performed on fetal spleen and lung tissues by direct ELISA (ELISA BVD/MD antigen mix screening, Pourquier) following manufacturer instructions to assess the presence of BVD virus.

\section{Molecular identification and characterization}

Molecular identification and characterization was performed on isolated fungal mycelia. Genomic DNA extraction, PCR and RFLP were performed as described by Machouart and coll. [17]. Specific sense primers for Rhizopus spp., Rhizomucor spp., Mucor spp., and Lichtheimia corymbifera (RpL1，5' TGATCTACGTGACAAATTCT 3'; RmL1, 5' TGATCTACGCGAGCGAACAA 3'; MucL1, 5' TGATCTACGTGACATATTCT 3'; and AbsL1, 5' TGATCTACACGGCATCAAAT 3', respectively) were used with a degenerate antisense primer (MR1, 5' AGTAGTTTGTCTTCGGKCAA 3'). The region selected for the design of primers excluded the amplification of human DNA and other filamentous fungi. Primers Lap (5' GAAACTGCGAATGGCTCATTA 3') and Rap (5' CAATCCAAGAATTTCACCTCT 3') designed to amplify all the fungi and the human DNA were used as positive controls.

The amplicon was also sub-cloned into the $2886 \mathrm{bp}$ pTZ57R/T vector (Fermentas, Glen Burnie, USA) and subjected to RFLP analysis with AclI restriction enzyme.

\section{Results}

\section{Macroscopic appearance of the fetus and placenta}

Cotyledons appeared thickened, firm and necrotic, whereas intercotyledonary chorioallantois showed brown exudate and moderate hyperemia (Fig. 1A). Multifocal and coalescing white, raised and dry plaques were present in the skin of the fetus, mainly localized on the head (periorbital regions) and on the back (Fig. 1B). Small, white, raised plaques were occasionally seen also in the skin of the abdomen, legs and tail. No gross lesions were present in the other organs.

\section{Finding of fungal hyphae in tissues sections}

Microscopically, mild necrotizing placentitis and vasculitis, mainly localized on the cotyledons, were evident in the placenta and in the larger vessels at the base of the cotyledons. Numerous fungal hyphae morphologically con-
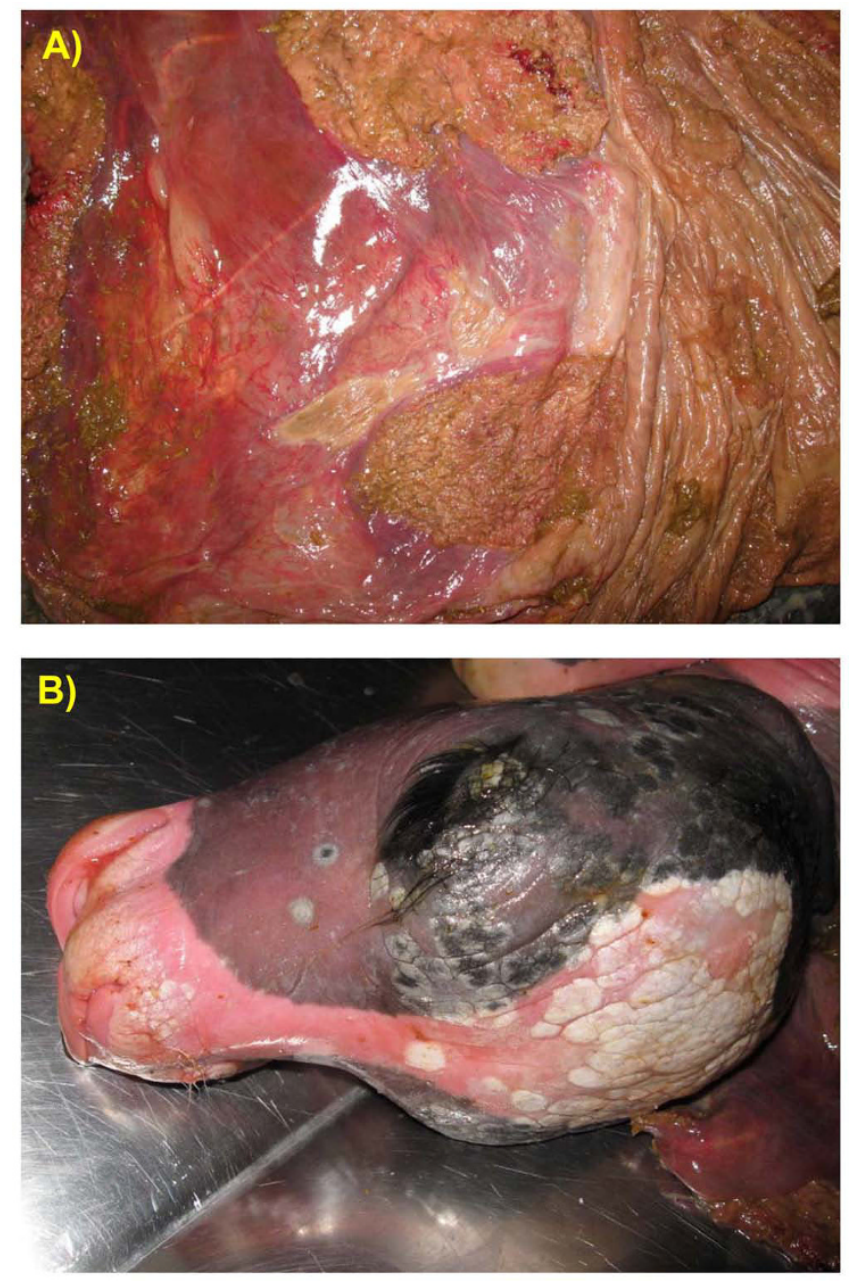

Figure I

Gross lesions on the fetus and placenta. A) The placenta is thickened, cotyledons appear necrotic and firm. Hyperemia of intercotyledonar areas is also visible. B) Aborted calf. White and irregular lesions localized in the head (periorbital region) are visible in the picture.

sistent with Zygomycetes were present in the grossly evident placentar lesions (Fig. 2A and 2B). Hyphae were elongate, filamentous, 6 to $15 \mu \mathrm{m}$ in diameter and strongly PAS and GMS positive. Skin lesions were characterized by spread multifocal areas of orthokeratotic hyperkeratosis, oedema and rare infiltrate of lymphocytes in the superficial derma.

Rare single hyphae were also present within the skin lesions. No other relevant alterations were detected in the other organs.

\section{Isolation of fungi}

Non-haemolytic, non-pathogenic Escherichia coli has grown up from liver, spleen, kidney, heart and lung. After 6 days, these plates were still negative for Brucella spp. 

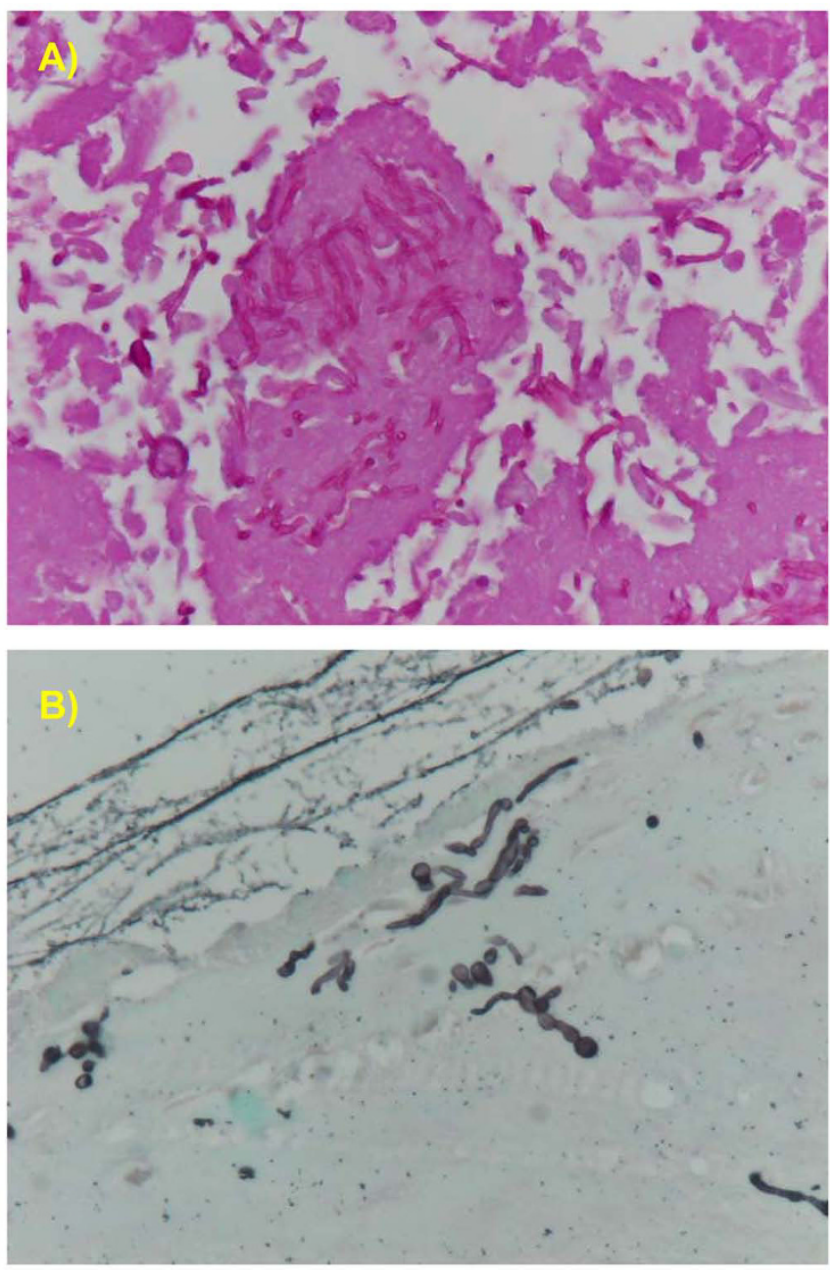

Figure 2

Histological findings on tissue sections. Histological section of the placenta. A) Numerous PAS positive hyphae are present within the necrotic cotyledons (PAS reaction, magnification $\times 200$ ). B) Numerous fungal hyphae (black), irregular in diameter and without regular septation, are present. (GMS stain, magnification $\times 200$ ).

growth. Colonies with typical morphological features of fungi grew up on Sabouraud agar after 24 hours of aerobic incubation from fetal skin and placenta. Colonies were fast growing, white at first becoming pale grey with age, and up to $1.5 \mathrm{~cm}$ high (Fig. 3A), woolly to cottony in aspect. The reverse side was uncoloured and without pigment production. On the colonies a direct microscopic examination with Cotton Blue staining showed a large number of nonseptate hyphae, with a diameter of 6 to 15 $\mu \mathrm{m}$. Specialised hyphae sporangiophores, hyaline or faintly pigmented in colour and simple or branched, arised in groups of three at the internodes (Fig. 3C). Sporangiophores showed relatively small sporangia (20-120 $\mu \mathrm{m}$ of diameter), typically pyriform in shape with characteristic conical-shaped columella and pronounced apoph- ysis (Fig. 3B), often with a short projection at the top, morphologically consistent with Lichtheimia corymbifera $[18,19]$. Sporangiospores were ellipsoid, light grey coloured and smooth-walled (Fig. 3D). They were detected in the sporangium and released to the surrounding area. The presence of rhizoids was not observed. Moreover, the isolate was not capable to utilize melezitose as sole carbon source.

Regarding the serological and virological results, all the blood samples were negative for antibodies against Leptospira serovars tested, BoHV-1, Brucella abortus and Chlamydophila abortus. The mother of the examined fetus was seropositive at low titre for BVDV antibodies. One of the other aborted dams was seropositive for Neospora caninum and the third one was seropositive for BVDV, Neospora caninum and Coxiella burnetii. The serological analyses on the fetal blood sample were negative for the abortive agents tested. The ELISA performed on fetal tissues for BVD virus gave negative result.

\section{Molecular identification and characterization of the fungal isolate}

An expected specific amplicon of 824 bp was obtained only with the set of primers specifying for Lichtheimia (ex Absidia) corymbifera. In contrast none amplification products were obtained when primers for Rhizopus spp., Rhizomucor spp. and Mucor spp. were applied (Fig. 4A). The 824 bp amplicon was sub-cloned into pTZ57R/T vector and both the 824 bp PCR amplicon (Fig. 4B) and the subcloned amplicon (Fig. 4C) were analyzed by RFLP with AclI restriction enzyme. Acll digestion generated 2 restriction fragments of 518 and $306 \mathrm{bp}$ for the $824 \mathrm{bp}$ PCR amplicon and 3 fragments of 1846, 1481 and 373 bp for the subcloned 824 bp amplicon. In both cases the restriction pattern corresponds to Lichtheimia corymbifera (Fig. 4D). Sequencing of the $824 \mathrm{bp}$ cloned fragment further confirmed the specificity of the amplicon and the absence of hypothetical polymorphisms or mutations.

\section{Discussion}

In the area where the abortions occurred, the nutrition of dairy cattle is regulated by strict rules and the administration of silage is forbidden. Consequently, dairy cows are fed with a major amount of hay. In our case, heifers were usually fed with grass and hay, while during the pregnancy the ration was supplemented with hay stored the year before in a moist place, exposed to bad weather conditions. The risk factors associated to the administration of mouldy hay were present. Moreover, the macroscopic lesions on the fetus and placenta were consistent with mycotic abortion, confirmed by cultural and histological findings [20]. The morphological aspect of the colonies on Sabouraud agar, the microscopic aspect of the hyphae, sporangia and sporangiospores and the execution of the 

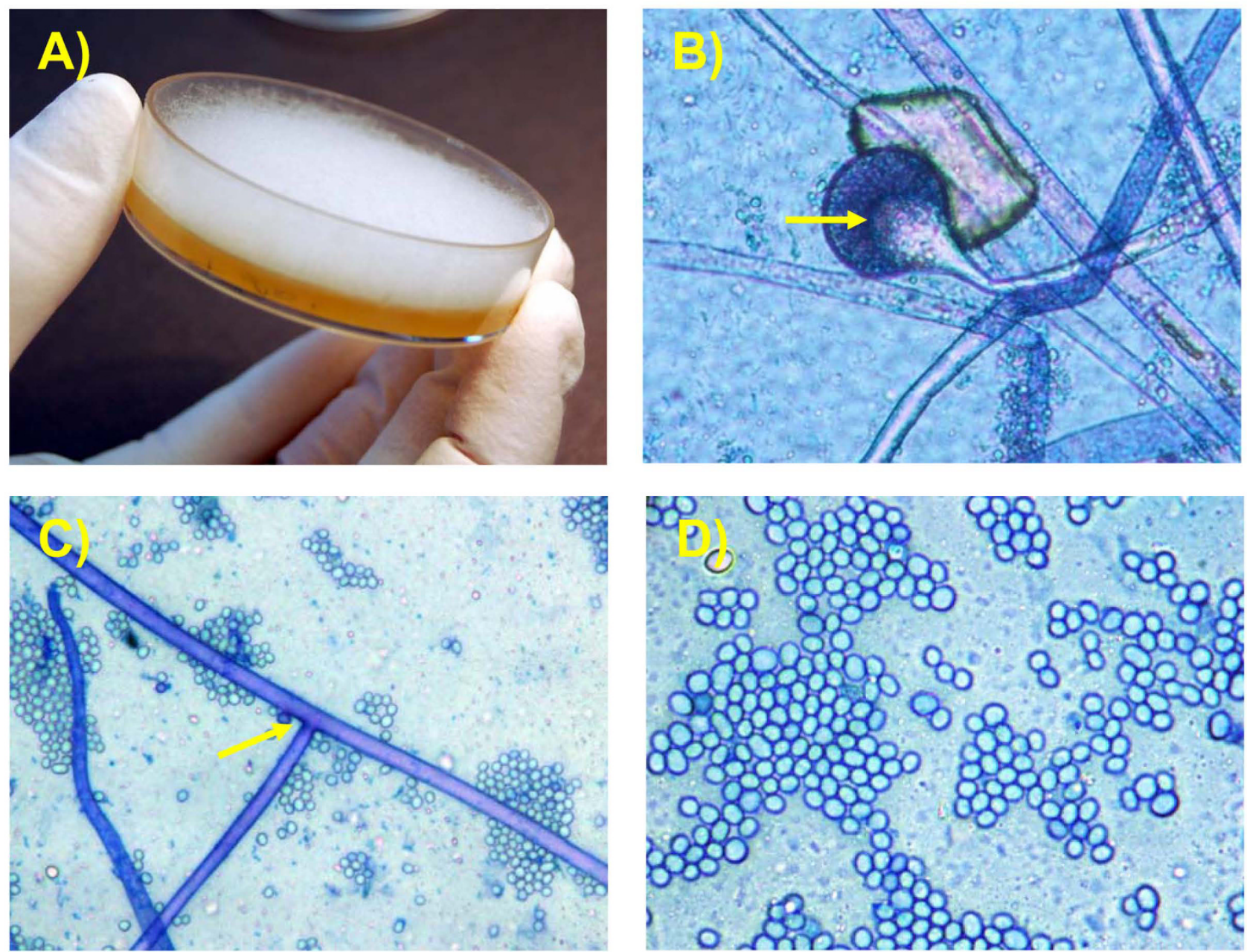

Figure 3

Cultural and microscopic aspect of the fungus. A) Fungal culture. The whitish, woolly or cottony aspect of the colonies is evident. The colony raises $1.5 \mathrm{~cm}$ high. B) Microscopic aspect of sporangia, pyriform with a conical-shaped columella (indicated by arrow) and pronounced apophysis (Cotton Blue stain, magnification $\times 400)$. C) Nonseptate hyphae arising in groups of three at the internode (arrow, magnification $\times 400)$. D) Ellipsoidal sporangiospores (magnification $\times 1000)$.

PCR and RFLP directed the diagnosis to Lichtheimia corymbifera $[17,18]$. The isolation of non-pathogenic $E$. coli from fetal tissues, associated to the absence of gross lesions in the fetal organs, and the BVDV seropositivity at low titre of the dam have to be considered not meaningful to demonstrate the involvement of these pathogens in the abortive event [21]. In addition, necrotizing placentitis is rarely associated to BVDV, and in our case BVD virus was not detected by direct diagnostic procedures on fetal spleen and lungs.

\section{Conclusion}

Our findings support the conclusion that Lichtheimia corymbifera is the causative agent of the abortion. The present case is particularly relevant because represents the first isolation in Italy of Lichtheimia corymbifera in a bovine aborted fetus. It is useful to stress that in case of abortion the routine diagnostic procedures should include the mycotic component and assess the fungal species [4]. In accordance to the assumption that mycotic abortion is characterised by the presence of mycotic elements associated with placentitis and fetal dermatitis, and that morphologic characteristics of hyphae in tissues have to be compatible with cultured isolates $[18,19]$, confirmation lies in the isolation and identification of the specific mycotic agent. In our case, identification has been confirmed by PCR, and the characterization of the isolate has been made by RFLP. This diagnostic procedure allowed to establish without doubt the etiology of abortion. 

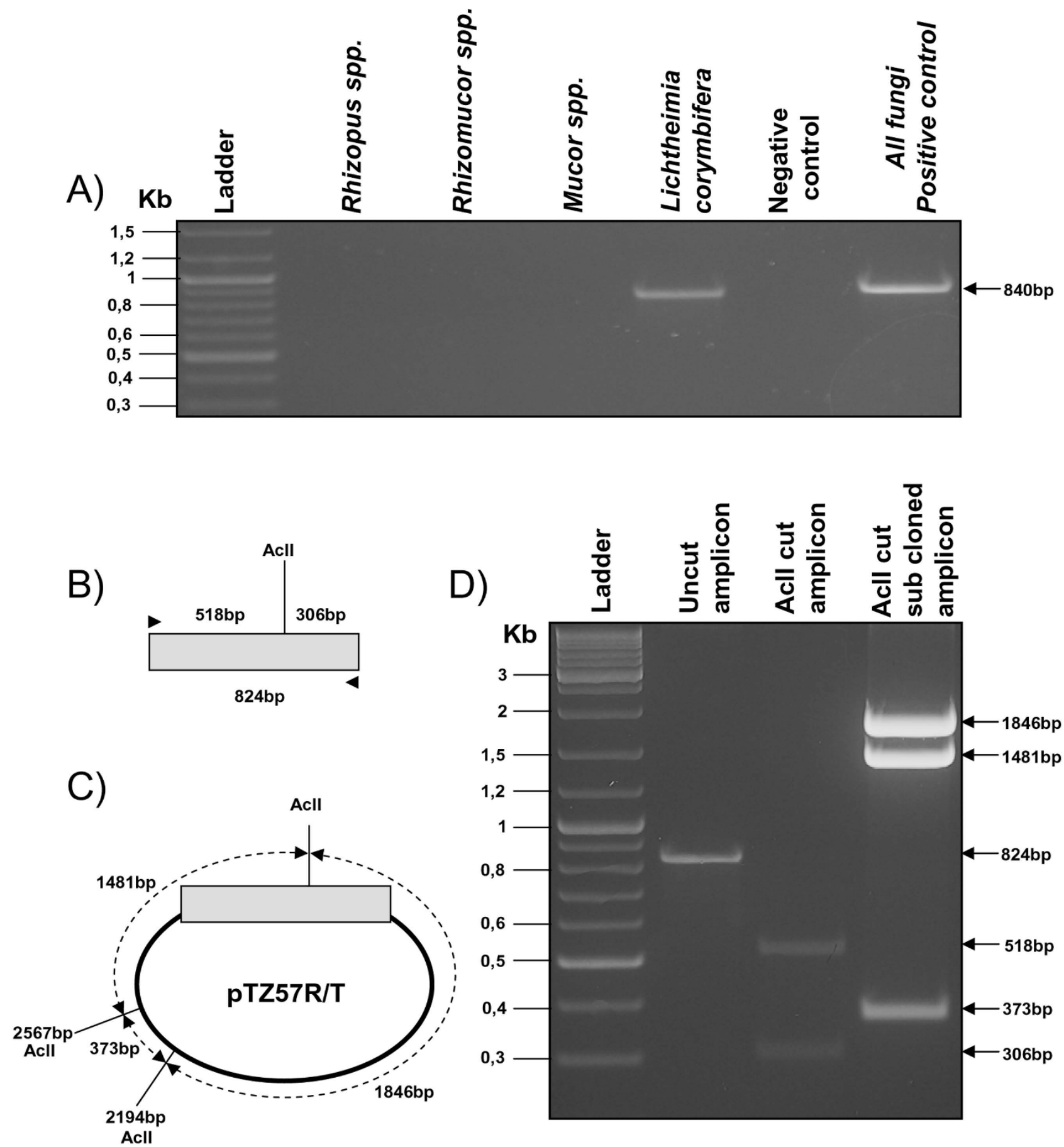

\section{Figure 4}

Molecular characterization of Lichtheimia corymbifera. A) Electrophoresis patterns of amplicons obtained with specific sets of primers for Lichtheimia corymbifera, Rhizopus spp., Rhizomucor spp., Mucor spp. and a set of primers for all other filamentous fungi used as a positive control (840 bp). B) Diagram showing the amplicon (not on scale) and the Acll restriction enzyme predicted fragments of 518 and 306 bp. C) Diagram showing the sub-cloned 824 bp amplicon (not on scale) into the pTZ57R/ $\mathrm{T}$ vector and Acll restriction sites within the vector (2194 bp and $2567 \mathrm{bp}$ ) and within the amplicon. D) Electrophoresis patterns of Acll uncut 824 bp amplicon, Acll cut 824 bp amplicon and Acll cut sub-cloned 824 bp amplicon. 


\section{Competing interests}

The authors declare that they have no competing interests.

\section{Authors' contributions}

CSC conceived, designed and wrote the paper. CP and FG performed the bacteriological, mycological and serological tests. SJ performed the histological tests. GD was responsible for the molecular identification and characterisation, and intellectually contributed and helped to write the paper. ST performed the serological tests and intellectually contributed and helped to write the paper. $\mathrm{SC}$ reviewed the paper. All authors read and approved the final manuscript.

\section{References}

I. Committee on Bovine Reproductive Nomenclature: Recommendations for Standardizing Bovine Reproduction Terms. Cornell Vet 1972, 62:216-237.

2. Anderson $M L$ : Infectious causes of bovine abortion during midto late-gestation. Theriogenology 2007, 68:474-486.

3. Knudtson WU, Kirkbride CA: Fungi associated with bovine abortion in the northern plains states (USA). J Vet Diagn Invest 1992, 4: $181-185$

4. Ali R, Khan IH: Mycotic abortion in cattle. Pak Vet J 2006, 26:44-46.

5. Givens MD, Marley SMD: Infectious causes of embryonic and fetal mortality. Theriogenology 2008, 70:270-285.

6. Johnson CT, Lupson GR, Lawrence KE: The bovine placentome in bacterial and mycotic abortions. Vet Rec 1994, 12:263-266.

7. Chihaya Y, Furusawa Y, Okada H, Matsukawa K, Matsui Y: Pathological Studies on Systemic Mycoses in calves. J Vet Med Sci 1991 53:105।-1058.

8. Quinn PJ, Carter ME, Markey B, Carter GR: The pathogenic zygomycetes. In Clinical Veterinary Microbiology WOLFE Publishing: |994:409-4|4

9. Walker RL: Mycotic bovine abortion. In Current therapy in large animal theriogenology Edited by: Youngquist RS, Threlfall WR. St. Louis, Elsevier; 2007:417-419.

10. Carter ME, Cordes DO, Mennea ME, Hunter R: Fungi Isolated from Bovine Mycotic Abortion and Pneumonia with Specia Reference to Mortierella wolfii. Res Vet Sci 1973, 14:20I-206.

II. McCausland IP, Slee KJ, Hirst FS: Mycotic abortion in cattle. Aust Vet J 1987, 64: I29-1 32.

12. Di Menna ME, Carter ME, Cordes DO: The identification of Mortierella wolfii isolated from cases of abortion and pneumonia in cattle and a search for its infection source. Res Vet Sci 1972, 13:439-442.

13. Hugh-Jones ME, Austwick PCK: Epidemiological studies in bovine mycotic abortion. I. The effect of climate on incidence. Vet Rec 1967, 85:273.

14. MacDonald SM, Corbel M]: Mortierella wolfii infection in cattle in Britain. Vet Rec 1981, 7:419-421.

15. Cavirani S, Foni E, Ferrari A, Donofrio G, Cabassi CS, Scatozza F: Infectious causes of bovine abortion in northern Italy dairy herds. XX World Buiatric Congress, Sydney 6-10 1998, 2:1 II6.

16. Schwarz P, Lortholary O, Dromer F, Dannaoui E: Carbon assimilation profiles as a tool for identification of Zygomiycetes. J Clin Microbiol 2007, 45: I433-1439.

17. Machouart M, Larché J, Burton K, Collomb J, Maurer P, Cintrat A, Biava MF, Greciano S, Kuijpers FA, Contet-Audonneau N, De Hoog GS, Gérard A, Fortier B: Genetic identification of the main opportunistic Mucorales by PCR-restriction fragment length polymorphism. J Clin Microbiol 2006, 44:805-8I0.

18. Garcia-Hermoso D, Hoinard D, Gantier JC, Grenouillet F, Dromer F, Dannaoui E: Molecular and phenotypic evaluation of Lichtheimia corymbifera (ex Absidia corymbifera) complex isolates associated with human mycosis: rehabilitation of $L$. ramosa, ex synonym of $\mathbf{L}$. corymbifera. / Clin Microbiol 2009 in press.

19. Hoffmann K, Discher S, Voigt K: Revision of genus Absidia (Mucorales, Zygomycetes) based on physiological, phylogenetic and morphological characters; thermotolerant Absidia spp. form a coherent group, Mycocladiacae fam. nov. Mycol Res 2007, III:1 | 69-1 I83.

20. Schlafer DH, Miller RB: Female genital system. In Jubb, Kennedy and Palmer's Pathology of Domestic Animals Edited by: Grant Maxie M. Saunders Elsevier; 2007:429-564.

21. Brock KV, Grooms DL, Givens MD: Reproductive Disease and Persistent Infections. In Bovine Viral Diarrhea Virus. Diagnosis, management and control Edited by: Goyal SM, Ridpath JF. Blackwell Publishing, lowa; 2005: 145-I56.
Publish with Bio Med Central and every scientist can read your work free of charge

"BioMed Central will be the most significant development for disseminating the results of biomedical research in our lifetime. "

Sir Paul Nurse, Cancer Research UK

Your research papers will be:

- available free of charge to the entire biomedical community

- peer reviewed and published immediately upon acceptance

- cited in PubMed and archived on PubMed Central

- yours - you keep the copyright
BioMedcentral 